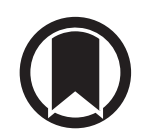

CrossMark

\title{
Confronting the controversy: interleukin-6 and the COVID-19 cytokine storm syndrome
}

\author{
Luke Y.C. Chen $\mathbb{1}^{1,2}$, Ryan L. Hoiland $\mathbb{1}^{3,4}$, Sophie Stukas ${ }^{5}$, \\ Cheryl L. Wellington ${ }^{5}$ and Mypinder S. Sekhon $\mathbb{1}^{6}$
}

Affiliations: 'Division of Hematology, Dept of Medicine, University of British Columbia, Vancouver, BC, Canada. ${ }^{2}$ Centre for Health Education Scholarship, University of British Columbia, Vancouver, BC, Canada. ${ }^{3}$ Dept of Anesthesiology, Pharmacology and Therapeutics, University of British Columbia, Vancouver, BC, Canada. ${ }^{4}$ Dept of Cellular and Physiological Sciences, Faculty of Medicine, University of British Columbia, Vancouver, BC, Canada. ${ }^{5}$ Dept of Pathology and Laboratory Medicine, International Collaboration on Repair Discoveries, School of Biomedical Engineering, Djavad Mowafaghian Centre for Brain Health, Faculty of Medicine, University of British Columbia, Vancouver, BC, Canada. ${ }^{6}$ Division of Critical Care Medicine, Dept of Medicine, University of British Columbia, Vancouver, BC, Canada.

Correspondence: Luke Y.C. Chen, 2775 Laurel St, 10th Floor, Vancouver, BC, V5Z1M9, Canada. E-mail: Ichen2abccancer.bc.ca

@ERSpublications

Hypercytokinaemic immune dysregulation in COVID-19 is known as cytokine storm syndrome. Interleukin-6 levels $\geqslant 80 \mathrm{pg} \cdot \mathrm{mL}^{-1}$ predict an increased risk of respiratory failure and death, and immunomodulatory therapy is an area of urgent investigation. https://bit.ly/3jq3uJ4

Cite this article as: Chen LYC, Hoiland RL, Stukas S, et al. Confronting the controversy: interleukin- 6 and the COVID-19 cytokine storm syndrome. Eur Respir J 2020; 56: 2003006 [https://doi.org/10.1183/ 13993003.03006-2020].

\section{Introduction}

The concept of coronavirus disease 2019 (COVID-19)-related cytokine storm syndrome (COVID-CSS) emerged early in the severe acute respiratory syndrome coronavirus 2 (SARS-CoV-2) pandemic to explain why some patients exposed to this virus become critically ill with acute respiratory distress syndrome, multi-organ failure, and death. A seminal study from Wuhan, China reported higher serum concentrations of inflammatory cytokines in patients requiring critical care compared to those with milder disease, and the authors postulated that "cytokine storm was associated with disease severity" [1]. COVID-19 hypercytokinaemia initially invited comparisons to other respiratory viral infections that cause a dysregulated immune response, namely severe acute respiratory syndrome (SARS) and Middle East respiratory syndrome (MERS). Subsequently, similarities between COVID-CSS and other cytokine storm syndromes such as haemophagocytic lymphohistiocytosis (HLH) [2], autoinflammatory syndromes, and chimeric antigen T-cell therapy cytokine release syndrome (CAR T-cell CRS) became apparent [3-5]. The proposition that hypercytokinaemia is pathological in some patients with COVID-19 catalysed numerous clinical trials of immunomodulatory and cytokine-inhibitor therapy. However, critics contend that CSS is a misleading conceptual framework in COVID-19 and two prominent editorials have raised significant doubt about COVID-CSS [6, 7]. In brief, these authors contend that:

- there is no definition of cytokine storm syndrome;

- median IL-6 levels are relatively low in COVID-19; 
- COVID-19 should be characterised as a hypoinflammatory vasculopathy rather than a hyperinflammatory hypercytokinaemia syndrome;

- therefore, immunomodulatory therapy may play little or no role in the treatment of COVID-19.

In this editorial, we address these controversies and demonstrate that cytokine storm syndrome may play an important role in severe respiratory failure and mortality caused by COVID- 19 .

\section{Definition of cytokine storm syndrome}

Critics of the COVID-CSS concept have claimed that "cytokine storm has no definition" [6], and that there is "no evidence that COVID patients develop a cytokine storm" [7]. In fact, experts define cytokine storm syndrome as a clinical phenotype of [8]:

- immune dysregulation characterised by perpetuated activation of lymphocytes and macrophages;

- resulting in secretion of large quantities of cytokines;

- leading to overwhelming systemic inflammation and multi-organ failure with high mortality.

The term CSS was first coined to describe the hypercytokinaemia in graft versus host disease after allogeneic stem cell transplant [9]. More recently, CAR T-cell CRS, which exhibits markedly elevated IL-6 levels often in excess of $1000 \mathrm{pg} \cdot \mathrm{mL}^{-1}$ (normal $<7 \mathrm{pg} \cdot \mathrm{mL}^{-1}$ ), and responds to IL- 6 blockade, has been included in the umbrella term CSS [3]. Many viral, bacterial and parasitic infections can cause CSS. Infectious pathogens such as Epstein-Barr virus (EBV) and Mycobacterium tuberculosis cause pathological immune activation characterised by markedly elevated cytokines and cytokine receptors such as interferon- $\gamma(\mathrm{IFN}-\gamma)$ and soluble IL-2 receptor (sIL-2r) in patients with inherited and acquired immune defects, leading to the clinical syndrome of HLH $[10,11]$.

Detailed immunological studies have clearly established that some patients with severe COVID-19 indeed meet the general criteria for cytokine storm syndrome in a manner which is unique from other infectious CSS. A recent longitudinal comparison in patients with moderate versus severe COVID-19 demonstrated three distinct signatures of "immunological misfiring" in those with severe disease [5]. Patients with moderate disease (patients admitted to hospital who survived and did not require intensive care unit (ICU) admission) had low expression of inflammatory cytokines and increased tissue reparative growth factors, such as epidermal growth factor, platelet-derived growth factor and vascular endothelial growth factor (figure 1a). In contrast, patients with severe disease (those who died or required ICU admission) had highly elevated pro-inflammatory cytokines including IL- $1 \alpha$, IL- $1 \beta$, IL-6, IL-18 and TNF- $\alpha$ (figure $1 \mathrm{~b}$ ) [5]. Another study found that, whereas bacterial sepsis-induced immunoparalysis is characterised by monocyte deficiency and inability to produce cytokines, in severe COVID-19, peripheral blood mononuclear cells exhibit sustained TNF- $\alpha$ and IL-6 production with LPS stimulation ex-vivo and markedly elevated IL-6 and C-reactive protein (CRP) concentrations in vivo [4]. When compared to other respiratory viruses such as SARS-CoV-1, MERS-CoV and human parainfluenza virus and respiratory syncytial virus, the transcriptional hyperinflammatory response in SARS-CoV-2 is uniquely imbalanced, with low IFN-I and -III levels, accompanied by very high expression of inflammatory chemokines and cytokines (IL-1, IL-6) and severe lymphopenia, resulting in a "high pro-inflammatory, low innate antiviral defence" state [12].

Although there are not yet standard diagnostic criteria for COVID-CSS, the term is generally used to denote the subset of patients who demonstrate excessive immune activation characterised by markedly elevated inflammatory markers such as CRP, IL-6 and ferritin, as well as lymphopenia and a spectrum of end organ damage from isolated respiratory dysfunction to multi-organ failure. Clinical measurement of IL-6 is not widely available at present but is a relatively simple and inexpensive assay for many clinical laboratories to implement. In the early days of COVID-19 in our own centre, when IL- 6 was being measured for research purposes rather than clinical care, we used the following criteria for COVID-CSS: 1) COVID-19 pneumonia requiring mechanical ventilation; 2) fever (maximum temperature $>38^{\circ} \mathrm{C}$ ); 3 ) $\mathrm{CRP}$ $>100 \mathrm{mg} \cdot \mathrm{L}^{-1}$; and 4) peak serum ferritin $>1000 \mu \mathrm{g} \cdot \mathrm{L}^{-1}$ [13]. Patients meeting these criteria had markedly elevated research serum IL-6 levels: median $91 \mathrm{pg} \cdot \mathrm{mL}^{-1}$, interquartile range (IQR) $54-696 \mathrm{pg} \cdot \mathrm{mL}^{-1}$ $(n=15)$. However, IL-6 does not always correspond to CRP (which is produced by hepatocytes in response to IL-6) or ferritin (which is a marker of macrophage activity) [14]. Recently, at least two large studies have shown that serum IL-6 is superior to CRP, ferritin, liver enzymes and other simple clinical laboratory markers for predicting clinical outcomes, such as respiratory failure and death, with an optimal cut-off of 80 and $86 \mathrm{pg} \cdot \mathrm{L}^{-1}$, respectively $[15,16]$. Taken together, these data imply that an IL-6 threshold $\geqslant 80 \mathrm{pg} \cdot \mathrm{mL}^{-1}$ carries prognostic value in future definitions of COVID-CSS. Ultimately, multivariate prognostic models incorporating simple, widely available parameters, such as peripheral oxygen saturation $\left(\mathrm{S}_{\mathrm{pO}_{2}}\right)$ /inspired oxygen fraction $\left(F_{\mathrm{IO}_{2}}\right)$, neutrophil/lymphocyte ratio, CRP and ferritin, 

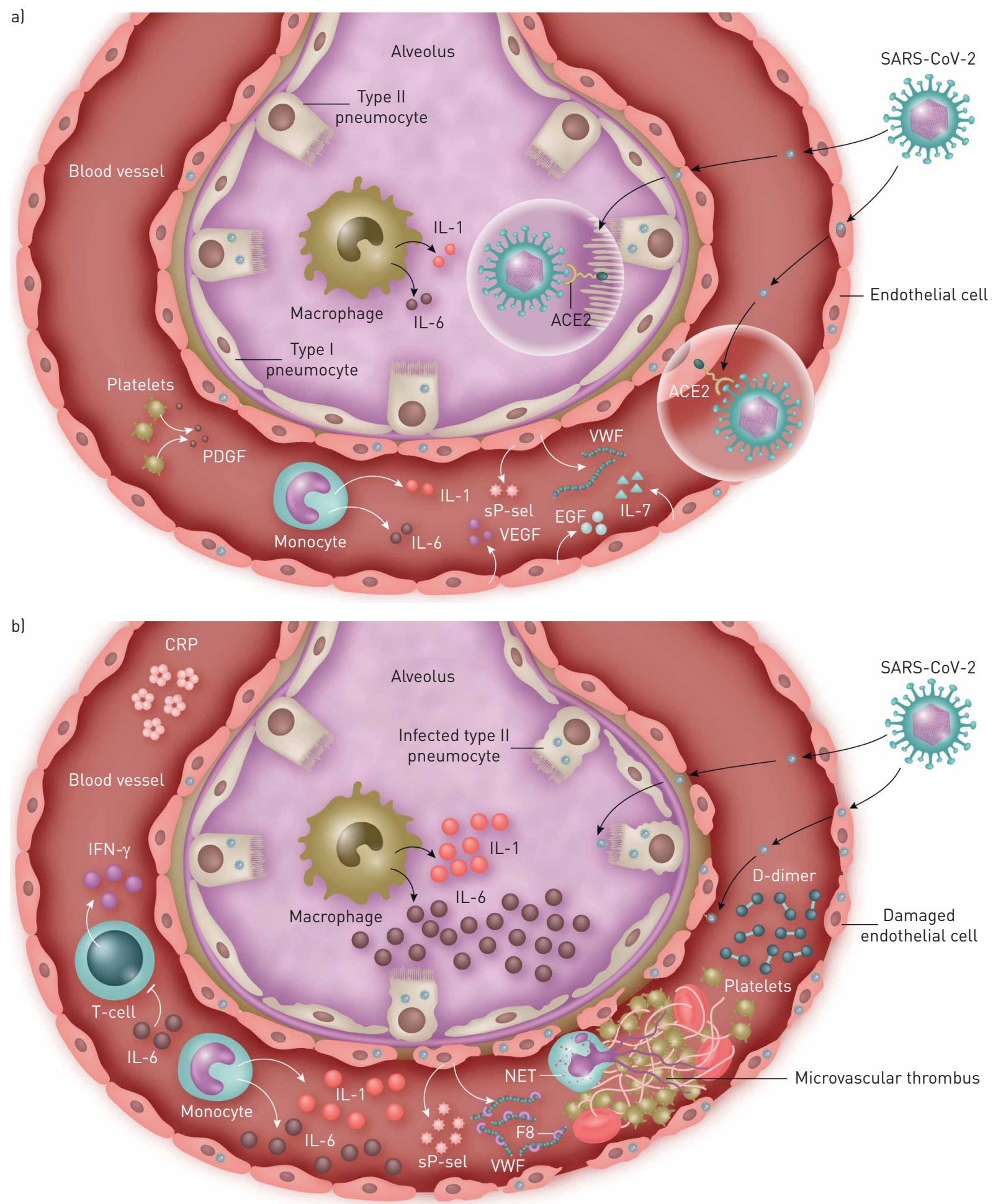

FIGURE 1 Schematic representation of mechanisms by which the novel severe acute respiratory syndrome coronavirus 2 (SARS-CoV-2) causes mild disease in some patients and severe disease in others. In both cases, the virus enters human cells via binding to angiotensin-converting enzyme 2 (ACE2), a transmembrane receptor widely expressed in type II pneumocytes, macrophages, endothelial and other pulmonary cells [24, 25]. a) Mild disease (low incidence of coagulopathy and thrombosis, shorter hospital stay, low critical illness and mortality): these patients have low serum inflammatory cytokines and high tissue reparative growth factors such as epidermal growth factor (EGF), platelet derived growth factor (PDGF), and interleukin (IL)-7 [5]. VEGF: vascular endothelial growth factor. b) Severe disease (high incidence of micro- and macrovascular thrombosis, longer hospital stay, high critical illness, and mortality): many of these patients have cytokine storm, with high serum inflammatory cytokines (such as IL-6, IL-1, IFN- $\gamma$ ) and markers of endothelial activation such as von Willebrand factor (vWF), factor 8 coagulant (F8) and soluble P-selectin (sP-sel), resulting in endothelialitis and microvascular thrombosis [5, 15, 27]. Markedly elevated IL-6 leads to lymphopenia and immunoparalysis which is partially restored by IL-6 blockade [4, 32]. NET: neutrophil extracellular trap; CRP: C-reactive protein. 
along with cytokines/chemokines such as IL-1, IL-6, IFN- $\gamma$-induced protein (IP)-10 and IL-10, may lead to prognostic scoring systems rather than the binary "yes/no" diagnostic model employed in HLH $[16,17]$.

\section{Interleukin-6 and COVID-CSS}

The modest serum IL-6 elevations reported in early studies of COVID-19 have been cited as an argument against the relevance of CSS in this disease $[6,7]$. For example, the median $\left(7-45 \mathrm{pg} \cdot \mathrm{mL}^{-1}\right)$ and IQR for
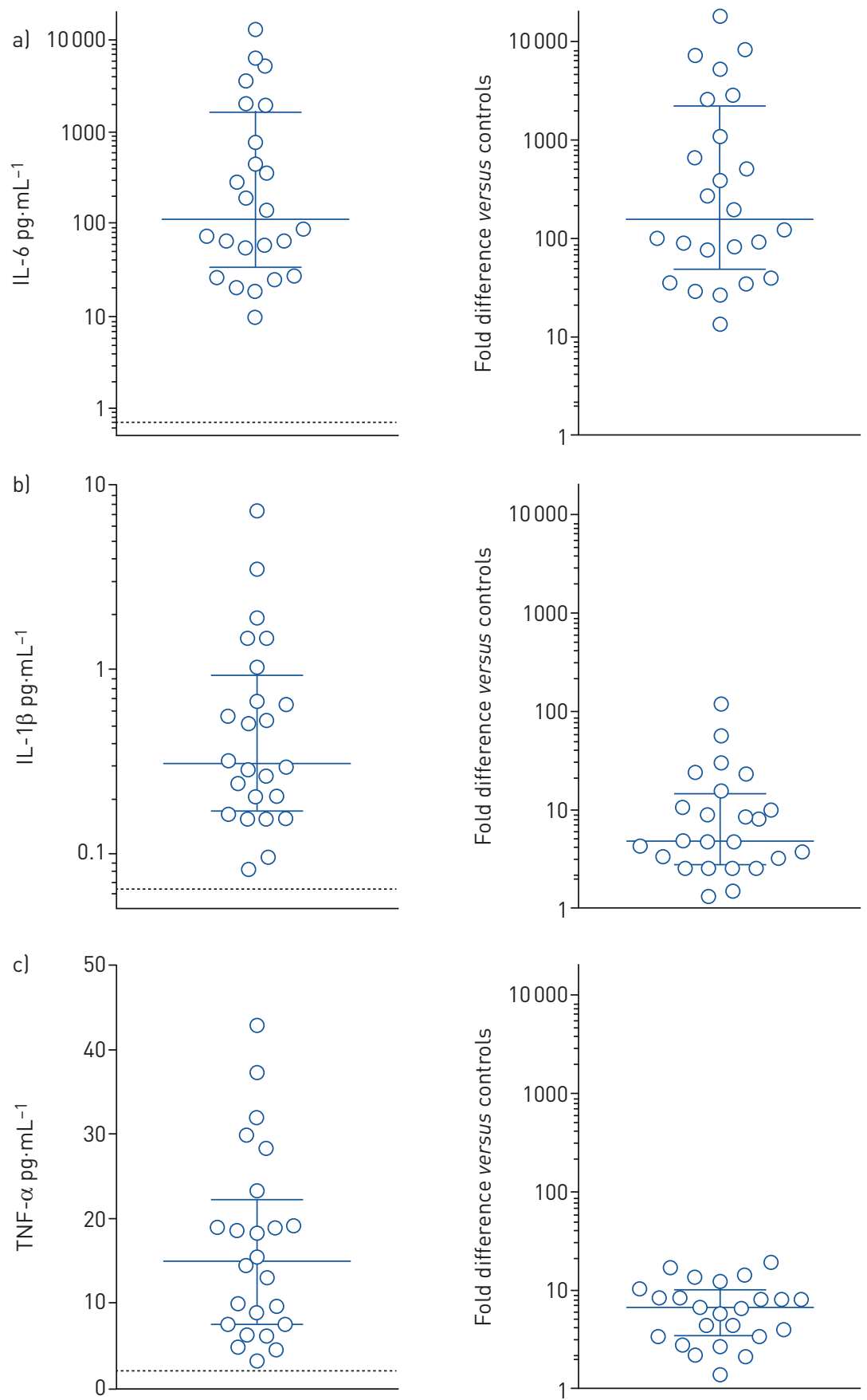

FIGURE 2 Absolute cytokine levels and their elevation compared to normative values. Depicted are the peak levels for a) interleukin (IL)-6, b) IL-1 $\beta$ and c) tumour necrosis factor (TNF)- $\alpha$ from a Vancouver cohort of 24 critically ill coronavirus disease 2019 (COVID-19) patients. Further, we have represented the corresponding fold difference between cytokine levels observed in the COVID-19 patients and those of healthy controls li.e. normative values) on the right-sided figure for each cytokine. These normative values can be seen as a horizontal dashed line for IL-6 (value $0.71 \mathrm{pg} \cdot \mathrm{mL}^{-1}$ ), IL-1 $\beta$ (value $0.064 \mathrm{pg} \cdot \mathrm{mL}^{-1}$ ) and TNF- $\alpha$ (value $2.23 \mathrm{pg} \cdot \mathrm{mL}^{-1}$ ). All values were derived from the Simoa $\mathrm{HD}-1$ analyser $[13,33]$. 
serum IL-6 levels were relatively low in four large studies of patients with mild to severe COVID-19 [6]. However, median and IQR are estimates of central tendency, and in cytokine storm, the population of interest resides within the top quartile of patients. Further, the temporal heterogeneity of cytokine analysis in these studies may have missed rapid increases in cytokine levels in some patients. Importantly, cytokine storm pertains not to the majority of patients who exhibit a physiological response to a given exposure, but to the minority who suffer from pathological immune activation or dysfunction. To provide some analogies, only a minute proportion of those exposed to EBV develop HLH, and less than 15\% of patients undergoing CAR T-cell therapy develop severe grade 3-4 CRS requiring IL-6 blockade. Likewise, a small but highly relevant proportion of those exposed to COVID-19 develop CSS.

In COVID-CSS, many inflammatory cytokines, such as IL-1, IL-10 and TNF- $\alpha$ are elevated approximately 2-100 fold above normative values, whereas IL- 6 demonstrates much larger increases, in some cases more than 1000 fold above normal. Figure 2 illustrates levels of inflammatory cytokines IL-1 $\beta$, IL- 6 and TNF- $\alpha$ in 24 patients with severe COVID-19 requiring ICU admission in our centre, both in absolute quantities and as a "fold elevation" above normative values. Several other large studies have reported markedly elevated serum IL-6 levels in the 100-10000 pg.mL ${ }^{-1}$ range in patients with severe disease $[12,15,17,18]$. These markedly elevated IL-6 levels in COVID-CSS are similar in magnitude to severe CAR T-cell CRS [19], and higher than other hyper-IL-6 syndromes, such as multicentric Castleman disease, where IL-6 is elevated but typically $<100 \mathrm{pg} \cdot \mathrm{mL}^{-1}[3,20]$. HLH is said to encompass a diverse spectrum of "hyperferritinaemic hyperinflammatory syndromes with a common terminal pathway but with different pathogenetic roots" [21]. Likewise in COVID-CSS, it appears increasingly likely that diverse "root" signatures of immune dysregulation lead to a common terminal hyperinflammatory pathway characterised by markedly elevated IL-6, severe T-cell lymphopenia, and respiratory failure $[4,5,17]$.

\section{Hypercytokinaemia, vasculopathy and endothelialitis}

Some authors have proposed that the markedly elevated D-dimer levels and high rates of micro- and macrovascular thrombosis, in contrast to modestly elevated median IL-6 levels, signify that COVID-19 pathophysiological sequelae are related to hypoinflammatory vasculopathy rather than inflammatory cytokine storm [7]. The central role of the receptor angiotensin-converting enzyme 2 (ACE2) in viral cell entry and the complex vascular changes, including intussusceptive angiogenesis and diffuse endothelial membrane disruption seen in autopsy studies, indeed support a central role for vasculopathy in COVID-19-related morbidity [22]. However, recent studies have demonstrated that hypercytokinaemia and vasculopathy are not an either/or proposition. The pulmonary vasculature is a key site of intravascular coagulopathy and thrombotic complications of COVID-19 [23, 24]. Earlier studies of the SARS-CoV spike (S) protein and ACE2 demonstrated that binding of the $S$ protein to ACE2 results in production of inflammatory cytokines, including IL-1 and IL-6 $[25,26]$.

This hyperinflammatory response results in disruption of the endothelial barrier and hypercoagulability. Diffuse occlusion in the microcirculation of the pulmonary vascular bed may cause pulmonary hypertension, increased dead space ventilation and, ultimately, right heart failure. Serum endothelial factors, such as soluble thrombomodulin, soluble P-selectin and von Willebrand factor play a major role in disease progression to acute respiratory distress syndrome [27]. Taken together, these findings suggest a model in which COVID-19 is best viewed as an inflammatory endothelialitis, with direct viral infection of pneumocytes, endothelial and epithelial cells producing inflammatory cytokines and immune-mediated damage to the vasculature and surrounding tissue $[25,27]$ (figure $1 \mathrm{~b}$ ).

\section{Cytokines, respiratory failure and immunomodulatory therapy in COVID-19}

Critics of CSS contend that hypercytokinaemia seen in COVID-19 may be a necessary physiological response for viral clearance and warn against the use of immunomodulatory therapy in this context $[6,7]$. In the more established CSS, the central role of particular cytokines is determined not only by studies linking cytokine elevation to characteristic organ damage, but also clinical trials demonstrating that cytokine blockade results in clinical benefit. Primary HLH is perhaps the paradigm for this model, in that the IFN- $\gamma /$ CXCL-9 axis is central to the pathophysiology of the disease [28], and blocking this axis with emapalumab produces deep and sustained remissions [29]. Likewise, IL-1 has been shown to play a similar role in autoinflammatory syndromes and secondary $\mathrm{HLH}$, and blockade with anakinra or canakinumab results in clinical benefit $[30,31]$.

Evidence that IL-6 drives immune dysregulation and respiratory failure in COVID-CSS is rapidly accumulating. Elevated serum IL-6 is associated with lymphopenia, impaired lymphocyte cytotoxicity, and endothelial activation (figure $1 \mathrm{~b}$ ). These immune defects can be partially restored by treatment with IL- 6 blockade with tocilizumab $[4,32]$. A German study demonstrated that IL- $6>80 \mathrm{pg} \cdot \mathrm{mL}^{-1}$ in combination with CRP $>97 \mathrm{mg} \cdot \mathrm{L}^{-1}$ is highly predictive of respiratory failure [15]. In our own centre, IL-6 was found to 
be inversely related to the ratio of arterial oxygen tension to $F_{\mathrm{IO}_{2}}$ and static lung compliance [33]. Moreover, a multivariate model generated from a large prospective cohort $(n=501)$ showed that early systemic inflammation predicts mortality. Among 15 variables measured at hospital admission, CRP at a cut-off of $87.5 \mathrm{mg} \cdot \mathrm{L}^{-1}$ was the most sensitive $(0.97)$ and IL-6 at a cut-off of $86 \mathrm{pg} \cdot \mathrm{mL}^{-1}$ was the most specific (0.89) for predicting death [16].

The RECOVERY trial demonstrating mortality benefit from treatment with dexamethasone $6 \mathrm{mg}$ daily, particularly in patients with severe disease, is the first randomised controlled trial (RCT) to definitively show benefit for immunomodulatory therapy [34]. Although numerous retrospective studies of cytokine blockade, such as anakinra and tocilizumab, and JAK inhibition, such as baricitinib, have shown promise, ultimately RCTs are needed. Several of these studies are underway or recently completed, and the results are eagerly anticipated [3]. COVID-19 patients demonstrating the CSS phenotype are certainly on the severe end of the disease spectrum. Therefore, trials should aim to target patients with a dysregulated immune response to ascertain the true efficacy of immunomodulatory therapies. On 29 July, 2020, a press release for the COVACTA RCT (NCT04320615) comparing tocilizumab and placebo in COVID-19 reported no difference in the primary outcomes (clinical status and mortality). However, there were positive trends in duration of hospital stay and ventilator-free days, not considered significant because of the failure to meet the primary end-points. The inclusion criteria for the COVACTA trial were quite broad, requiring only a definite diagnosis of COVID-19 pneumonia with an oxygen saturation of $93 \%$ or lower or $S_{\mathrm{pO}_{2}} / F_{\mathrm{IO}_{2}}<300 \mathrm{mmHg}$, with no mention of markers of systemic inflammation such as CRP or IL-6. Given the gradient seen towards more benefit in severely ill patients with dexamethasone treatment in the RECOVERY trial, subgroup analysis in the COVACTA trial, as well as ongoing immunomodulatory RCTs, will be of great interest.

Acknowledgement: The authors thank Catherine Biggs for her critical review of the manuscript.

Author contributions: All authors contributed to the conception and drafting of the article and provided critical revision for important intellectual content and final approval.

Conflict of interest: None declared.

Support statement: L.Y.C. Chen is supported by the UBC Hematology Research Program. M.S. Sekhon is supported by the Vancouver Coastal Health Research Institute Clinician Scientist Award. C.L. Wellington is supported by grants from Canadian Institutes for Health Research, National Institutes of Health, Weston Brain Institute and Cure Alzheimer Fund. Funding information for this article has been deposited with the Crossref Funder Registry.

\section{References}

1 Huang C, Wang Y, Li X, et al. Clinical features of patients infected with 2019 novel coronavirus in Wuhan, China. Lancet 2020; 395: 497-506.

2 Mehta P, McAuley DF, Brown M, et al. COVID-19: consider cytokine storm syndromes and immunosuppression. Lancet 2020; 395: 1033-1034.

3 England JT, Abdulla A, Biggs CM, et al. Weathering the COVID-19 storm: lessons from hematologic cytokine syndromes. Blood Rev 2020; in press [https://doi.org/10.1016/j.blre.2020.100707].

4 Giamarellos-Bourboulis EJ, Netea MG, Rovina N, et al. Complex immune dysregulation in COVID-19 patients with severe respiratory failure. Cell Host Microbe 2020; 27: 992-1000. e1003.

5 Lucas C, Wong P, Klein J, et al. Longitudinal analyses reveal immunological misfiring in severe COVID-19. Nature 2020; 584: 463-469.

6 Sinha P, Matthay MA, Calfee CS. Is a "cytokine storm" relevant to COVID-19? JAMA Intern Med 2020; 180: $1152-1154$.

7 Leisman DE, Deutschman CS, Legrand M. Facing COVID-19 in the ICU: vascular dysfunction, thrombosis, and dysregulated inflammation. Intensive Care Med 2020; 46: 1105-1108.

8 Shimizu M. Clinical Features of Cytokine Storm Syndrome. In: Cron RQ, Behrens EM, eds. Cytokine Storm Syndrome. Cham, Springer International Publishing, 2019; pp. 31-41.

9 Clark IA. The advent of the cytokine storm. Immunol Cell Biol 2007; 85: 271-273.

10 Hayden A, Park S, Giustini D, et al. Hemophagocytic syndromes (HPSs) including hemophagocytic lymphohistiocytosis (HLH) in adults: a systematic scoping review. Blood Rev 2016; 30: 411-420.

11 Hayden A, Lin M, Park S, et al. Soluble interleukin-2 receptor is a sensitive diagnostic test in adult HLH. Blood Adv 2017; 1: 2529-2534.

12 Blanco-Melo D, Nilsson-Payant BE, Liu WC, et al. Imbalanced host response to SARS-CoV-2 drives development of COVID-19. Cell 2020; 181: 1036-1045.

13 Hoiland RL, Stukas S, Cooper J, et al. Amelioration of COVID-19 related cytokine storm syndrome: parallels to chimeric antigen receptor-T cell cytokine release syndrome. Br J Haematol 2020; 190: e150-e154.

14 Chen LYC, Hayden A, Mattman A. Extreme hyperferritinaemia, soluble interleukin-2 receptor, and haemophagocytic lymphohistiocytosis. Br J Haematol 2019; 185: 605-606.

15 Herold T, Jurinovic V, Arnreich C, et al. Elevated levels of IL-6 and CRP predict the need for mechanical ventilation in COVID-19. J Allergy Clin Immunol 2020; 146: 128-136.e4.

16 Laguna-Goya R, Utrero-Rico A, Talayero P, et al. IL-6-based mortality risk model for hospitalized patients with COVID-19. J Allergy Clin Immunol 2020; in press [https://doi.org/10.1016/j.jaci.2020.07.009]. 
17 Laing AG, Lorenc A, Del Barrio ID, et al. A dynamic COVID-19 immune signature includes associations with poor prognosis. Nat Med 2020; in press [https://doi.org/10.1038/s41591-020-1038-6].

18 Price CC, Altice FL, Shyr Y, et al. Tocilizumab treatment for cytokine release syndrome in hospitalized COVID-19 patients: survival and clinical outcomes. Chest 2020; in press [https://doi.org/10.1016/j.chest.2020.06.006].

19 Hay KA, Hanafi LA, Li D, et al. Kinetics and biomarkers of severe cytokine release syndrome after CD19 chimeric antigen receptor-modified T-cell therapy. Blood 2017; 130: 2295-2306.

20 van Rhee F, Wong RS, Munshi N, et al. Siltuximab for multicentric Castleman's disease: a randomised, double-blind, placebo-controlled trial. Lancet Oncol 2014; 15: 966-974.

21 La Rosee $\mathrm{P}$, Horne $\mathrm{A}$, Hines $\mathrm{M}$, et al. Recommendations for the management of hemophagocytic lymphohistiocytosis in adults. Blood 2019; 133: 2465-2477.

22 Ackermann M, Verleden SE, Kuehnel M, et al. Pulmonary vascular endothelialitis, thrombosis, and angiogenesis in Covid-19. N Engl J Med 2020; 383: 120-128.

23 McGonagle D, O'Donnell JS, Sharif $\mathrm{K}$, et al. Immune mechanisms of pulmonary intravascular coagulopathy in COVID-19 pneumonia. Lancet Rheumatol 2020; 2: e437-e445.

24 Huertas A, Montani D, Savale L, et al. Endothelial cell dysfunction: a major player in SARS-CoV-2 infection (COVID-19)? Eur Respir J 2020; 56: 2001634.

25 Colling ME, Kanthi Y. COVID-19-associated coagulopathy: an exploration of mechanisms. Vasc Med 2020; in press [https://doi.org/10.1177/1358863X20932640].

26 He L, Ding Y, Zhang Q, et al. Expression of elevated levels of pro-inflammatory cytokines in SARS-CoV-infected ACE2+ cells in SARS patients: relation to the acute lung injury and pathogenesis of SARS. $J$ Pathol 2006; 210: 288-297.

27 Goshua G, Pine AB, Meizlish ML, et al. Endotheliopathy in COVID-19-associated coagulopathy: evidence from a single-centre, cross-sectional study. Lancet Haematol 2020; 7: e575-e582.

28 Zoller EE, Lykens JE, Terrell CE, et al. Hemophagocytosis causes a consumptive anemia of inflammation. J Exp Med 2011; 208: 1203-1214.

29 Locatelli F, Jordan MB, Allen C, et al. Emapalumab in children with primary hemophagocytic lymphohistiocytosis. N Engl J Med 2020; 382: 1811-1822.

30 de Koning HD, Schalkwijk J, van der Ven-Jongekrijg J, et al. Sustained efficacy of the monoclonal anti-interleukin-1 beta antibody canakinumab in a 9-month trial in Schnitzler's syndrome. Ann Rheum Dis 2013; 72: $1634-1638$

31 Mehta P, Cron RQ, Hartwell J, et al. Silencing the cytokine storm: the use of intravenous anakinra in haemophagocytic lymphohistiocytosis or macrophage activation syndrome. Lancet Rheumatol 2020; 2: e358-e367.

32 Mazzoni A, Salvati L, Maggi L, et al. Impaired immune cell cytotoxicity in severe COVID-19 is IL-6 dependent. J Clin Invest 2020; 130: 4694-4703.

33 Stukas S, Hoiland RL, Cooper J, et al. The association of inflammatory cytokines in the pulmonary pathophysiology of respiratory failure in critically ill patients with coronavirus disease 2019. Crit Care Explorations 2020; 2: $\mathrm{e} 0203$

34 Horby P, Lim WS, Emberson JR, et al. Dexamethasone in hospitalized patients with Covid-19 - preliminary report. N Engl J Med 2020; in press [https://doi.org/10.1056/NEJMoa2021436]. 